\title{
ESTRESSE OCUPACIONAL E SAÚDE MENTAL DOS PROFISSIONAIS DO CENTRO DE ESPECIALIDADES MÉDICAS DE ARACAJU
}

\author{
OCCUPATIONAL STRESS AND THE MENTAL HEALTH OF THE PROFESSIONALS OF THE MEDICAL \\ SPECIALTIES CENTER OF ARACAJU, SERGIPE, BRAZIL
}

\author{
ESTRÉS LABORAL Y SALUD MENTAL DE LOS PROFESIONALES DEL CENTRO DE ESPECIALIDADES \\ MÉDICAS DE ARACAJU, SERGIPE, BRASIL
}

\author{
Arthur Marcelino Filho ${ }^{1}$ \\ Tania Maria de Araújo ${ }^{2}$
}

Resumo As características ambientais e o modo de organização do trabalho em centros especializados em saúde podem produzir situações de estresse e efeitos negativos sobre a saúde dos trabalhadores. O objetivo do estudo foi avaliar a saúde mental dos profissionais de saúde da rede especializada de Aracaju (Sergipe) e as situações de estresse ocupacional com base no modelo esforço-recompensa. Estudo transversal realizado em 2011 com 94 profissionais de saúde em atividade em dois centros de atendimento especializado. Foi utilizado questionário contendo variáveis sociodemográficas e econômicas, situação e ambiente de trabalho, avaliação de estresse ocupacional (usando o Effort-Reward Imbalance) e o Self Reporting Questionaire para mensuração dos transtornos mentais comuns. A prevalência de transtornos mentais comuns foi elevada $(50,6 \%)$, sendo mais frequente em fonoaudiólogos, psicólogos, terapeutas ocupacionais e fisioterapeutas e entre aqueles que relataram estar insatisfeitos com a sua condição salarial e de trabalho. Todos os profissionais que referiram desequilíbrio entre esforços e recompensas no trabalho apresentaram transtornos mentais comuns. Em conclusão, foram identificadas características do trabalho desfavoráveis aos profissionais e ao desempenho das suas funções, além de elevadas prevalências de transtornos mentais comuns. Programas voltados à proteção, valorização e promoção à saúde dos trabalhadores devem ser implantados a fim de elevar a qualidade de vida no trabalho.

Palavras-chave estresse; centro de especialidades médicas; transtornos mentais; fatores psicossociais do trabalho.
Abstract The environmental characteristics and the way work is organized in specialized health centers can lead to stressful situations and have negative effects on the workers' health. The aim of the study was to evaluate the mental health of health professionals working at the specialized network of Aracaju, state of Sergipe, Brazil, and situations of occupational stress based on the effort-reward model. It is a cross-sectional study conducted in 2011 among 94 health professionals working at two specialized care centers. A questionnaire containing demographic and economic variables, the situation and working environment, occupational stress assessments (using Effort-Reward Imbalance) and a Self-Reporting Questionnaire were used to measure common mental disorders. There was a high prevalence of common mental disorders (50.6\%), which were more frequent among speech therapists, psychologists, occupational therapists and physical therapists, and among those who reported being dissatisfied with their pay and work conditions. All professionals who mentioned imbalance between their efforts and their rewards at work presented common mental disorders. In sum, working characteristics unfavorable to the professionals and to the performance of their duties were identified, as was a high prevalence of common mental disorders. Programs aimed at the protection, enhancement and promotion of the workers' health must be implemented in order to improve their quality of life at work.

Keywords stress; medical specialties center; mental disorders; psychosocial factors at work. 
A relação entre o estresse ocupacional e a saúde dos trabalhadores é tema de várias pesquisas nos últimos anos. A literatura existente tem focalizado, com relativa importância, os profissionais de saúde, destacando que o trabalho em unidades de saúde pode estar associado à produção de sofrimento. Os profissionais que atuam nesses locais convivem cotidianamente com a doença e a dor dos que recorrem aos seus serviços. Estudos têm analisado os elementos determinantes ou contribuintes para tal situação, possibilitando melhor compreensão das relações que se estabelecem entre o profissional de saúde, o seu ofício e o adoecimento.

O trabalho, ao longo dos anos, vem passando por diversas mudanças, dentre as quais se incluem a distribuição do poder e o desenvolvimento tecnológico que exige mão de obra e conhecimentos específicos para sua execução. Essas mudanças, embora ofereçam maior comodidade e facilidades para a execução de numerosas tarefas, estão, por sua vez, elevando as exigências laborais, o que tem sobrecarregado o trabalhador, colocando em risco a sua saúde e o seu bem-estar (Magalhães, 2006).

O profissional de saúde da rede especializada está submetido a circunstâncias de elevadas exigências do trabalho em saúde que o sobrecarregam e podem levar ao adoecimento. A assistência na rede especializada é o ponto de estrangulamento nos serviços do Sistema Único de Saúde (SUS), em que os fluxos das unidades básicas são quase sempre conduzidos para as unidades especializadas, gerando longas filas para variados procedimentos e volume excessivo de trabalho (Franco e Magalhães Jr., 2004). Tais características criam tensões no ambiente de saúde, tanto para os que buscam atendimento especializado quanto para os que trabalham nesses locais.

Em um contexto como esse, em que as demandas externas excedem a capacidade do organismo humano, impossibilitando a sua reação, é que surge o estresse. O prolongamento do estresse pode implicar efeitos negativos sobre a saúde do indivíduo, tais como hipertensão arterial, depressão e ansiedade (Araújo et al., 2003). Assim, em razão da relevância desses problemas de saúde, os estudos sobre estresse e a saúde do trabalhador vêm ganhando importância na comunidade científica. Tais estudos consideram elementos multicausais do adoecer, identificando a interação de fatores patogênicos, com destaque para os fatores ambientais.

Entre os métodos científicos utilizados para avaliação da relação estresse e saúde, destacam-se aqueles que incorporam a análise de aspectos psicossociais do ambiente de trabalho. O desequilíbrio entre o esforço despendido pelo trabalho e o baixo reconhecimento por esse esforço é gerador de situações estressantes, o que pode ocasionar problemas de saúde (Griep et al., 2002). De acordo com o modelo esforço-recompensa, distintas tarefas devem ser realizadas mediante a troca de recompensa (dinheiro, autoestima, reconhecimento, perspectivas de carreira e segurança no trabalho). O mo- 
delo prediz que a falta de reciprocidade entre esforço despendido e a recompensa recebida desencadeia reações de estresse com consequências para a saúde física e mental do trabalhador (Siegrist et al., 2004; Chor et al., 2008; Souza et al., 2009; Von Dem Knesebeck et al., 2010; Nakao, 2010). Análise de fatores psicossociais no trabalho pode ser considerada importante medida para aferir as condições de saúde.

Apesar de significativa produção científica sobre o trabalho dos que cuidam da saúde, ainda são restritos os estudos que descrevem o estresse em diferentes categorias dos centros de saúde especializados (Machado e Assunção, 2012). Os estudos relacionados aos transtornos psíquicos em profissionais de saúde têm privilegiado as categorias dos médicos e dos enfermeiros, sobretudo em atividades hospitalares, mais especificamente na urgência e na emergência (Araújo et al., 2003; Fogaça et al., 2009; Souza et al., 2011; Cabana et al., 2012; Machado e Assunção, 2012). Adicionalmente, os estudos existentes pouco enfatizam a relação entre o esforço e a recompensa na condição de saúde física e mental do trabalhador da saúde na rede especializada ( $\mathrm{Li}$ et al., 2005; Vasconcelos e Guimarães, 2009).

Portanto, a investigação das condições de trabalho e de saúde mental das diversas categoriais ocupacionais poderá contribuir para melhor compreensão das características do trabalho produtoras de estresse nesse setor e das possíveis relações estabelecidas com as condições de saúde mental dos profissionais.

Este estudo tem o objetivo de descrever situações de esforço e de recompensas no trabalho na rede especializada de Aracaju (Sergipe), fatores que podem gerar estresse e sua relação com a saúde mental dos trabalhadores de modo a contribuir para o conhecimento da situação existente. A produção de um diagnóstico sobre a situação poderá ajudar na elaboração de medidas para proteger a saúde dos profissionais e, consequentemente, elevar a qualidade do atendimento prestado.

Pesquisas como esta podem ser úteis na sensibilização dos responsáveis pelas decisões políticas ou administrativas da cidade e do estado, voltadas para a promoção da saúde dos trabalhadores da saúde, contribuindo para a melhoria das condições de trabalho, a diminuição de faltas e licenças pelas condições do adoecimento e do estresse ocupacional. De acordo com Areias e Comandule (2006), a elevada frequência do estresse ocupacional tem levado ao adoecimento do trabalhador, gerando aumento dos custos dos serviços de saúde decorrente da baixa produtividade, constantes afastamentos e absenteísmo.

\section{Materiais e métodos}

Foi realizado um estudo epidemiológico de corte transversal com o intuito de descrever e analisar as condições de estresse ocupacional em profissionais e 
estimar a prevalência de transtornos mentais de dois centros de atenção especializados de Aracaju, estado de Sergipe: o Centro de Especialidades Médicas (Cemar) e o Centro de Especialidades Odontológicas (CEO). Os estudos de corte transversal são importantes métodos para identificar a prevalência de doenças na população. Esse tipo de estudo apresenta baixo custo, alto potencial descritivo, simplicidade analítica, rapidez, objetividade na coleta dos dados, facilidade de obter amostra representativa da população.

O estudo foi desenvolvido no Cemar, no bairro Siqueira Campos, em Aracaju. Diante do número reduzido de odontólogos nessa unidade, os profissionais do Centro de Especialidades Odontológicas (CEO), localizado no Cemar do bairro Augusto Franco, também participaram da pesquisa. Tais centros de saúde atendem pacientes de todo o estado de Sergipe que necessitam de atendimento clínico especializado.

Todos os trabalhadores de saúde de nível superior dessas duas unidades de atendimento foram considerados elegíveis para o estudo, totalizando 177. Portanto, foi realizado um censo dos trabalhadores desses serviços. Do total, 94 responderam ao questionário da pesquisa, distribuídos da seguinte forma: 26 médicos, 18 enfermeiros, dez odontólogos, nove assistentes sociais, oito fisioterapeutas, seis fonoaudiólogos, cinco psicólogos, cinco terapeutas ocupacionais, três nutricionistas, dois farmacêuticos e dois biomédicos.

Os profissionais foram contatados no próprio local de trabalho pelo pesquisador responsável, quando se solicitaram o preenchimento do questionário e a assinatura do termo de consentimento livre e esclarecido. Sempre que possível, o questionário foi preenchido nesse contato inicial, ou se agendou outro momento para a sua coleta.

Para a realização da pesquisa, elaborou-se questionário estruturado, contendo questões sobre aspectos sociodemográficos e econômicos, características do trabalho, avaliação do estresse ocupacional, utilizando o modelo Effort Reward Imbalance (ERI) (Siegrist et al., 2004); e da saúde mental, usando o Self-Reporting Questionnaire (SRQ-20) (Santos et al., 2009).

As características sociodemográficas e econômicas avaliadas foram as seguintes: sexo, idade, situação conjugal, filhos, cor da pele, escolaridade, ocupação, formação acadêmica, tempo de experiência profissional e renda média mensal.

Os aspectos do trabalho que foram avaliadas incluíram: elementos gerais (profissão, tipo de vínculo, jornada semanal, outros vínculos profissionais e nível de satisfação), ambiente (avaliação de ventilação, temperatura, iluminação, equipamentos de proteção, estrutura e recursos disponíveis para execução do serviço) e exigências (possibilidade de diálogo, carga emocional envolvida e presença de conflito entre tarefas demandadas).

As características do estresse ocupacional foram avaliadas considerando os pressupostos teóricos e metodológicos do ERI, um instrumento composto por 23 questões, utilizado como indicador do estresse ocupacional por meio de ava- 
liação dos esforços e recompensas no trabalho. É formado por três escalas: esforço (seis itens), recompensa (11 itens) e excessivo comprometimento com o trabalho (seis itens).

Nas escalas de esforço e de recompensa, as questões são construídas em dois níveis de resposta. Inicialmente, é solicitado que o trabalhador informe se concorda ou não com o enunciado da questão. Em seguida, solicita-se resposta com relação ao nível de estresse vivenciado com relação ao enunciado, com as seguintes opções de respostas: $1=$ nem um pouco estressado; 2 = pouco estressado; $3=$ estressado; e $4=$ muito estressado. A escala de comprometimento excessivo com o trabalho é medida segundo quatro níveis de resposta: $1=$ Discordo fortemente; $2=$ Discordo; $3=$ Concordo; $4=$ Concordo fortemente (Fernandez-Lopez et al., 2005; Chor et al., 2008; Souza et al., 2009; Siegrist, 2010).

As dimensões esforço, recompensa e comprometimento excessivo foram descritas em alto e baixo esforço, alta e baixa recompensa e alto e baixo comprometimento, adotando-se o ponto de corte na média do somatório de cada uma dessas dimensões para sua dicotomização. A razão desequilíbrio esforço-recompensa foi calculada por meio da equação: $(\Sigma \mathrm{E} \div[\Sigma \mathrm{R} \times \mathrm{c}])$, sendo $\mathrm{E}=$ esforço extrínseco e $\mathrm{R}=$ recompensa, multiplicada pelo fator de correção $(c=0,545455)$. Valores inferiores a 1 indicam uma condição favorável (situação de equilíbrio entre esforços e recompensas), e superiores a 1 indicam maior esforço gasto e menor recompensa recebida (Siegrist, 2010).

Avaliação da saúde foi feita utilizando-se o Self-Reporting Questionaire (SRQ-20). O SRQ foi desenvolvido pela Organização Mundial da Saúde (OMS) como instrumento de rastreamento de sintomas neuróticos, avaliando sintomas vivenciados nos últimos trinta dias. Consiste de vinte itens autoaplicáveis com respostas dicotômicas (sim/não), variando, portanto, de zero a 20. Um escore total é obtido pela soma dos pontos que confirmam a presença do sintoma a partir de um ponto de corte determinado (De Marco et al., 2008; Santos et al., 2009).

A validação do SRQ-20 para a realidade brasileira realizada por Mari e Willians (1986) evidenciou sensibilidade de $83 \%$ e especificidade de $80 \%$, com ponto de corte $7 / 8$. Alguns estudos têm adotado critério de escore maior que sete como critério de suspeição de caso, o que é indicativo da presença de sintomas psiquiátricos menores (De Marco et al., 2008). Neste estudo foi utilizado o ponto de corte de sete ou mais respostas positivas, como adotado em outros estudos com profissionais de saúde (Araújo et al., 2003; Nascimento Sobrinho et al., 2006).

\section{Análise dos dados}

Em consonância com os objetivos do estudo, a análise realizada foi do tipo descritiva, explorando as situações de estresse ocupacional e a frequência 
de transtornos mentais comuns na população estudada. Inicialmente procedeu-se à caracterização da população. Esta etapa incluiu aspectos sociodemográficos e econômicos considerando-se as variáveis do bloco de características sociodemográficas e do trabalho (idade, sexo, profissão, tempo de trabalho e renda média mensal, dentre outras).

Fatores estressores foram descritos de acordo com o modelo de desequilíbrio esforço-recompensa, detalhando-se os escores das escalas de esforço envolvidos nas tarefas e no nível de recompensa recebido. Avaliou-se também a razão entre essas duas escalas.

A prevalência global de transtornos mentais comuns (TMC) foi estimada na população estudada considerando-se o número de casos identificados (numerador) sobre o total de profissionais entrevistados em cada estrato analisado (denominador). Também foram calculadas as prevalências de TMC segundo as escalas do ERI, avaliando-se se o modelo é capaz de identificar situações de exposição à saúde mental dos profissionais de saúde da rede especializada de atenção de Aracaju.

Para a avaliação de associação bruta entre as dimensões do modelo esforço-recompensa e TMC, utilizou-se o teste qui-quadrado, estipulando valor de $\mathrm{p} \leqslant 0,05$ para associações estatisticamente significantes. Os dados foram armazenados e analisados com o uso dos programas estatísticos SPSS versão 17.0 e EpiInfo.

Este estudo foi submetido ao Comitê de Ética em Pesquisa do Instituto de Saúde Coletiva da Universidade Federal da Bahia, autorizado mediante o parecer n. 017-11/CEP-ISC. Os participantes da pesquisa assinaram o termo de consentimento livre e esclarecido de acordo com as portarias n. 196/1996 e n. 466/2012 do Ministério da Saúde. Os procedimentos do estudo foram desenvolvidos de forma a proteger a privacidade dos sujeitos, garantindo a participação anônima e voluntária.

\section{Resultados e discussão}

O Cemar e o CEO somavam, em conjunto, 177 profissionais. Destes, 152 eram do primeiro centro e 25 do segundo. Do total de 177, 94 profissionais $(53,1 \%)$ participaram da pesquisa, respondendo ao questionário. Nos serviços estudados, a categoria com maior número de profissionais foi a médica, seguida da dos enfermeiros. Os farmacêuticos e os biomédicos foram os profissionais em menor participação na população. Contudo, os médicos $(38,2 \%)$ e os dentistas $(40,0 \%)$ mostraram-se os profissionais com as menores taxas de adesão a esta pesquisa.

Quanto aos aspectos sociodemográficos, evidenciou-se predomínio de mulheres em relação aos homens nesses centros de especialidades. Outros 
estudos vêm demonstrando o aumento significativo da participação feminina na área da saúde (De Marco et al., 2008). A média de idade foi de 39,8 anos, portanto uma população relativamente jovem com maior proporção de indivíduos pardos (54,3\%), seguidos de brancos (34\%). Em relação à situação conjugal, a maioria era casada/união estável $(62,8 \%) ; 56,3 \%$ dos pesquisados possuíam filhos.

Os profissionais tinham atuação recente em suas carreiras. O tempo de término da formação superior mais frequente foi de seis a dez anos $(35,8 \%)$, e $79,8 \%$ possuíam título de especialistas. A busca por maior valorização salarial no mercado de trabalho tem levado os profissionais de saúde a recorrerem a especializações e residências (Nascimento Sobrinho et al., 2006). Os títulos de pós-graduação stricto sensu (mestrado e doutorado), no entanto, ainda foram restritos nas categorias pesquisadas. Os valores pecuniários atribuídos aos títulos de mestre e doutor são, respectivamente, de $12 \%$ e $15 \%$ a incidir sobre o salário base da categoria de acordo com o artigo 25 da lei complementar n. 61 do município de Aracaju, que dispõe sobre os planos de cargos e salários. O investimento atribuído a uma formação stricto sensu e o retorno financeiro obtido parecem desfavoráveis ao profissional, o que pode se tornar um fator a ser revisado de modo a se incentivar a busca por maior qualificação desses profissionais.

Em relação aos aspectos econômicos, a renda média dos profissionais investigados foi de $\mathrm{R} \$ 3.500,00 ; 58,3 \%$ declararam estar insatisfeitos com seus honorários. Apenas 35,4\% dos pesquisados residiam em casa própria e 93,8\% possuíam automóvel. Em relação ao nível de satisfação salarial conforme a categoria profissional, observou-se que os psicólogos, os terapeutas ocupacionais e os nutricionistas apresentaram unanimidade quanto à insatisfação salarial $(100 \%)$, seguidos de fisioterapeutas $(87,5 \%)$, fonoaudiólogos $(83,3 \%)$, odontólogos (70\%) e médicos (52\%). A insatisfação salarial foi, portanto, um dado relevante na pesquisa.

Nascimento Sobrinho e colaboradores (2006), ao pesquisarem os médicos de Salvador (BA), identificaram que a remuneração foi relatada como satisfatória, apesar da sobrecarga de trabalho. Entretanto, registra-se que a remuneração desse grupo profissional é, em geral, mais elevada que a das demais categorias da saúde. Portanto, o nível de satisfação com relação a esse aspecto parece ser bem diferente quando se comparam os grupos por profissão.

Guimarães e colaboradores (2011), em sua pesquisa sobre insatisfação com o trabalho em profissionais em centros de atenção psicossocial, verificaram que a insatisfação com o trabalho emergia de causas como falta de materiais e estrutura, relações conflituosas com a equipe e gestores e as condições salariais. Siqueira e Pedruzzi (2009) identificaram que 92\% de uma amostra de quarenta fonoaudiólogos do estado de Alagoas possuíam 
remuneração incompatível com a sua formação. A ausência de organizações de classe de algumas categorias profissionais, como sindicatos e associações bem estruturados, pode ser um fator importante para a instabilidade profissional, os baixos salários e a precarização das condições de trabalho e de emprego. Tais fatores, por sua vez, podem contribuir para que os profissionais tenham uma dupla ou até tripla jornada de trabalho. O duplo emprego foi referido por grande parte dos pesquisados (84\%). Segundo a literatura, a manutenção de outro trabalho é também uma característica frequente de enfermeiros (Araújo et al., 2003), professores (Delcor et al., 2004) e médicos (Nascimento Sobrinho et al., 2006).

Considerando o tempo de trabalho no Centro de Especialidades Médicas de Aracaju, observou-se que os pesquisados trabalhavam há pouco tempo na unidade: 52,3\% tinham tempo de trabalho inferior a cinco anos, e 73,4\% haviam feito o ingresso no serviço por meio de concurso público municipal. A carga horária de trabalho de trinta horas semanais foi referida por $53,2 \%$. Um percentual expressivo de profissionais possuía outros vínculos de trabalho (84\%), sendo que $46,9 \%$ trabalhavam em outros serviços públicos de saúde no mesmo município ou em outros, por meio de concurso público estadual ou federal. Declararam-se insatisfeitos ou muito insatisfeitos com o local de trabalho 56,7\% dos entrevistados.

Ao especificar satisfação com o trabalho conforme a categoria profissional, verificou-se que psicólogos $(100 \%)$, fisioterapeutas $(75,0 \%)$, terapeutas ocupacionais $(75,0 \%)$, nutricionistas $(66,7 \%)$ e fonoaudiólogos $(60,0 \%)$ declararam-se totalmente insatisfeitos e insatisfeitos com o seu trabalho.

Quanto às condições do ambiente de trabalho, a precariedade dos equipamentos técnicos $(51,1 \%)$, a falta de equipamentos de proteção individual $(48,9 \%)$ e a insuficiência dos recursos gerais $(71,3 \%)$ foram aspectos assinalados com maior frequência pelos profissionais. Quesitos ligados ao bem-estar, tais como ventilação, iluminação e temperatura, foram descritos, predominantemente, como razoáveis ou satisfatórios. A relação entre as exigências do ofício e os recursos disponíveis foi considerada como muito ruim ou ruim por $43,6 \%$ dos entrevistados. De acordo com Leite (2005), mudanças ocorridas no trabalho nas últimas décadas têm gerado uma onda de regressão dos direitos trabalhistas e precarização, o que impacta direta e negativamente sobre as condições de trabalho.

A prevalência global de transtornos mentais comuns foi elevada, atingindo 50,6\% do grupo estudado. Quanto às respostas positivas ao SRQ-20, destacaram-se as referências a: nervosismo $(69,9 \%)$, dor de cabeça $(58,1 \%)$, cansaço constante $(57,0 \%)$ dormir mal $(50,5 \%)$, dificuldades para realizar tarefas $(49,5 \%)$, sensações desagradáveis no estômago $(47,8 \%)$, tristeza $(45,2 \%)$, má digestão $(38,7 \%)$ e sofrimento diário com o trabalho $(36,6 \%)$ (Tabela 1). Os profissionais de saúde dos centros especializados estudados 
apresentaram alta prevalência de TMC, sendo mais elevada que a encontrada em outras pesquisas com profissionais de saúde e que utilizaram o mesmo instrumento de mensuração para identificação de casos, o SRQ-20. Araújo e colaboradores (2003) identificaram a prevalência de distúrbios psíquicos menores de 33,3\% entre trabalhadores de enfermagem num hospital público de Salvador. No estudo de Nascimento Sobrinho e colaboradores (2006), encontrou-se uma prevalência de 26,0\% em profissionais médicos de Salvador.

Cabana e colaboradores (2007) assinalaram uma prevalência de TMC de 23,4\% em médicos no hospital geral da rede estadual de Recife (PE). No estudo de De Marco e colaboradores (2008), 15,8\% dos profissionais de saúde do Departamento de Psiquiatria da Universidade Federal de São Paulo apresentaram transtornos mentais comuns. Portanto, os dados encontrados na população estudada são bastante preocupantes e evidenciam a necessidade de atenção imediata da gestão de serviços.

Tabela 1

Frequência de respostas afirmativas para as perguntas do SRQ referidas por profissionais do Centro de Especialidades Médicas de Aracaju e Centro de Especialidades Odontológicas, Aracaju, Sergipe, Brasil, 2011

\begin{tabular}{|c|c|c|}
\hline Fatores do SRQ-20 & $\mathbf{n}$ & $\%$ \\
\hline \multicolumn{3}{|l|}{ Diminuição da energia } \\
\hline Trabalho diário causa sofrimento & 34 & 36,6 \\
\hline Dificuldades para realizar, com satisfação, as tarefas diárias & 46 & 49,5 \\
\hline Ter dificuldade para tomar decisões & 33 & 35,5 \\
\hline Cansa-se o tempo todo & 53 & 57,0 \\
\hline Ter dificuldade para pensar com clareza & 27 & 29,0 \\
\hline Cansa-se com facilidade & 48 & 51,6 \\
\hline \multicolumn{3}{|l|}{ Sintomas somáticos } \\
\hline Dormir mal & 47 & 50,5 \\
\hline Ter sensações desagradáveis de estômago & 44 & 47,8 \\
\hline Ter dores de cabeça & 54 & 58,1 \\
\hline Ter má digestão & 36 & 38,7 \\
\hline Ter tremores nas mãos & 11 & 11,8 \\
\hline Ter falta de apetite & 16 & 17,2 \\
\hline \multicolumn{3}{|l|}{ Humor depressivo/ansioso } \\
\hline Sente-se nervoso, tenso ou preocupado & 65 & 69,9 \\
\hline Ter se sentido triste ultimamente & 42 & 45,2 \\
\hline Assusta-se com facilidade & 33 & 35,5 \\
\hline Ter chorado mais do que de costume & 20 & 21,5 \\
\hline \multicolumn{3}{|l|}{ Pensamentos depressivos } \\
\hline Ter perdido o interesse pelas coisas & 22 & 22,9 \\
\hline Ser incapaz de desempenhar um papel útil na vida & 11 & 11,8 \\
\hline Sente-se uma pessoa inútil na vida & 9 & 9,7 \\
\hline Ter ideia de acabar com a vida & 2 & 2,2 \\
\hline
\end{tabular}

Fonte: Os autores. 
A análise da prevalência dos TMC segundo aspectos sociodemográficos identificou sua maior prevalência entre as mulheres (58\%) do que entre os homens $(25,0 \%)$; entre profissionais mais jovens e entre divorciados/separados/viúvos. Tais achados são semelhantes aos encontrados em outros estudos nacionais. Reis e colaboradores (2005), ao pesquisarem distúrbios psíquicos em professores da rede municipal de Vitória da Conquista (BA), encontraram uma prevalência de 55,9\% (57,2\% nas mulheres e $34 \%$ nos homens). Como possíveis explicações para esse achado, os estudos apontam as expressivas desvantagens para as mulheres no mercado de trabalho. As mulheres, mesmo que sejam trabalhadoras, ainda são as responsáveis pelo trabalho doméstico e cuidado da família, executando uma dupla jornada de trabalho. Esse aumento na carga de trabalho das mulheres faz com que elas exerçam, simultaneamente, os papéis de mãe, mulher, esposa e trabalhadora, elevando a carga total de trabalho (Araújo e Rotenberg, 2011).

Indivíduos com idade inferior a 30 anos foram os que apresentaram maior prevalência de TMC (62,5\%) (Tabela 2). Profissionais mais jovens estão em processo de entrada no mercado de trabalho. Tem-se verificado na literatura que os iniciantes, em geral, são os profissionais que recebem as tarefas mais penosas e difíceis, mas ainda não desenvolveram habilidades de enfrentamento satisfatórias para lidar com dificuldades e desafios do exercício profissional (Araújo et al., 2003). Esses fatores podem contribuir para maior prevalência de TMC, como observado neste estudo. Contudo, esse é um aspecto que deve ser levado em consideração em estudos futuros para sua melhor compreensão.

Araújo e colaboradores (2006), em estudo de base populacional com mulheres em cidade de médio porte na Bahia, também identificaram maior prevalência de TMC em viúvas/divorciadas (48\%). A perda do companheiro e as responsabilidades advindas de assumir a chefia da família sem a presença de apoio de outra pessoa foram fatores relacionados para explicar, ao menos em parte, esse achado.

O aumento da prevalência de TMC foi observado na medida em que diminuiu o nível de satisfação com o local de trabalho $(33,3 \%$ entre os muitos satisfeitos e 75,0\% entre os totalmente insatisfeitos). Situação similar foi encontrada com relação à satisfação com salários ou honorários (33,3\% entre os totalmente satisfeitos e 59,5\% entre os insatisfeitos). Esses dados corroboram a hipótese da presença de associação entre transtornos mentais comuns e satisfação com o salário e ambiente de trabalho entre os profissionais estudados. 
Tabela 2

Prevalência de TMC segundo aspectos socioeconômicos demográficos e condições do ambiente de trabalho dos profissionais do Centro de Especialidades Médicas de Aracaju e do Centro de Especialidades Odontológicas, Aracaju, Sergipe, Brasil, 2011

\begin{tabular}{|c|c|c|c|}
\hline \multirow{2}{*}{ Características (N) } & \multirow[t]{2}{*}{$\mathrm{N}$} & \multicolumn{2}{|c|}{ TMC } \\
\hline & & n & $\%$ \\
\hline Prevalência global & 89 & 45 & 50,6 \\
\hline Sexo (89) & & 5 & 25,0 \\
\hline Masculino & 20 & 40 & 58,0 \\
\hline Feminino & 69 & & \\
\hline Faixa etária (85) & 8 & 5 & 62,5 \\
\hline$<30$ anos & 27 & 13 & 48,1 \\
\hline 31 a 35 anos & 18 & 11 & 61,1 \\
\hline 36 a 40 anos & 13 & 7 & 53,8 \\
\hline 41 a 45 anos & 19 & 5 & 26,3 \\
\hline \multicolumn{4}{|l|}{$>45$ anos } \\
\hline Situação conjugal (88) & 22 & 9 & 40,9 \\
\hline Solteiro & 56 & 29 & 51,8 \\
\hline Casado/união estável & 10 & 7 & 70,0 \\
\hline \multicolumn{4}{|c|}{ Separado/divorciado/viúvo } \\
\hline \multicolumn{4}{|c|}{ Satisfação com o trabalho (85) } \\
\hline Totalmente insatisfeito & 8 & 6 & 75,0 \\
\hline Insatisfeito & 38 & 26 & 68,4 \\
\hline Satisfeito & 36 & 8 & 22,2 \\
\hline Muito satisfeito & 3 & 1 & 33,3 \\
\hline \multicolumn{4}{|c|}{ Satisfação com os honorários ( 88 ) } \\
\hline Totalmente satisfeito & 3 & 1 & 33,3 \\
\hline Parcialmente satisfeito & 33 & 13 & 39,4 \\
\hline Insatisfeito & 52 & 31 & 59,6 \\
\hline \multicolumn{4}{|l|}{ Tempo de trabalho (83) } \\
\hline Até 5 anos & 45 & 25 & 55,6 \\
\hline 5,01 a 10 anos & 29 & 14 & 48,3 \\
\hline 10,01 a 15 anos & 4 & 2 & 40,0 \\
\hline 15,01 a 25 anos & - & - & 0,0 \\
\hline Mais de 25 anos & 5 & 42 & 50,6 \\
\hline
\end{tabular}

Fonte: Os autores.

Os profissionais com tempo de trabalho menor que cinco anos $(55,6 \%)$ e aqueles com idade inferior a trinta $(62,5 \%)$ foram os que apresentaram as maiores prevalências de TMC (55,6\%) (Tabela 2$)$. O ofício em uma unidade de saúde especializada é ansiogênico e desencadeador de sofrimento aos que nesse ambiente trabalham. Entretanto, observou-se que quanto mais maduro era o indivíduo e quanto maior o tempo na unidade, menor era a prevalência de TMC. Tal fato pode estar indicando que os profissionais 
tendem a se adaptar às condições difíceis, criando estratégias que melhor os protegem; ou seja, com o tempo aprendem melhor a solucionar possíveis dificuldades ou mesmo conseguem ocupar situações de mais baixas exposições.

Além disso, as estratégias de coping são apontadas na literatura como mecanismos cognitivos e comportamentais que os indivíduos empregam diante de condições estressantes de trabalho (Sanzovo e Coelho, 2007). Cabe também considerar a possibilidade de que aqueles que se ajustam à realidade permanecem em suas funções. Por sua vez, os que não conseguem se adaptar podem ter saído, abandonado o emprego (viés de seleção). Além disso, é possível obter maiores possibilidades de escolhas para os profissionais que permanecem na instituição ao longo do tempo - o que permite que eles consigam mais vantagens, podendo se esquivar de postos de trabalho mais penosos (Reis et al., 2005).

Quanto à prevalência de TMC segundo a categoria profissional, foi observada maior ocorrência de TMC em profissionais de saúde cujo ofício associava-se às atribuições terapêuticas e de reabilitação, tais como: fonoaudiólogos $(83,3 \%)$, psicólogos $(80 \%)$, terapeutas ocupacionais (60\%) e fisioterapeutas $(57,1 \%)$. Além dessas categorias, 55,6\% dos enfermeiros, 50\% dos assistentes sociais e $44 \%$ dos médicos apresentaram TMC. Apenas $25 \%$ dos odontólogos apresentaram transtornos mentais comuns. Categorias como nutricionistas, farmacêuticos e biomédicos, pelo número reduzido de profissionais na unidade, não apresentaram amostra significativa para análise (Tabela 3).

\section{Tabela 3}

Prevalência de TMC segundo categoria profissional, Centro de Especialidades Médicas de Aracaju e Centro de Especialidades Odontológicas, Aracaju, Sergipe, Brasil, 2011

\begin{tabular}{lccc}
\hline & N & \multicolumn{2}{c}{ TMC } \\
Categoria profissional* & & $\mathbf{n}$ & $\%$ \\
\hline Médicos & 25 & 11 & 44,0 \\
Enfermeiros & 18 & 10 & 55,6 \\
Odontólogos & 8 & 2 & 25,0 \\
Assistentes sociais & 8 & 4 & 50,0 \\
Fisioterapeutas & 7 & 4 & 57,1 \\
Fonoaudiólogos & 6 & 5 & 83,3 \\
Psicólogos & 5 & 4 & 80,0 \\
Terapeutas ocupacionais & 5 & 3 & 60,0 \\
Nutricionistas & 3 & 1 & 33,3 \\
Farmacêuticos & 2 & - & 0,0 \\
Biomédicos & 2 & 1 & 50,0 \\
\hline Fonte: Os autores. & & & \\
* Para esta variável, foram perdidas informações (decorrente de SRQ-20 incompleto) para: um médico, dois odontólogos, \\
um fonoaudiólogo e um terapeuta ocupacional. & &
\end{tabular}


Na literatura, há ainda poucos estudos que referem TMC e estresse ocupacional nas categorias profissionais aqui analisadas. O aumento dos estudos sobre as condições de trabalho dessas categorias da saúde seria de grande contribuição para que fossem compreendidas as condições de trabalho desses profissionais.

De Marco e colaboradores (2008) identificaram uma prevalência de TMC de 37,5\% em assistentes sociais e de 14,4\% em terapeutas ocupacionais. O trabalho dos terapeutas ocupacionais e das demais categorias que focalizam suas atividades em ações terapêuticas tem como objetivo a reabilitação do indivíduo, realizando avaliação e tratamento de diversas disfunções mentais, comunicativas, motoras, sociais e de desenvolvimento global. A permanência contínua por tempo prolongado desses profissionais na reabilitação, a constante exposição às angústias e dificuldades dos pacientes, os processos lentos de obtenção de resultados em decorrência das características do tratamento - o qual depende, de forma mais acentuada do que em outras ocupações, da adesão do paciente - e o baixo reconhecimento profissional, especialmente observado com relação à remuneração, quando comparado a outras categorias profissionais nos centros estudados, podem ser fatores que explicam ao menos parcialmente tais achados.

Carvalho e Malagris (2007), ao avaliarem o nível de estresse em 31 profissionais de saúde em um posto de assistência médica, identificaram prevalência de estresse em $100 \%$ dos assistentes sociais e fonoaudiólogos. As outras categorias pesquisadas apresentaram as seguintes prevalências de estresse ocupacional: $75 \%$ dos enfermeiros, $67 \%$ dos odontólogos, $60 \%$ dos nutricionistas, $60 \%$ dos médicos e $50 \%$ dos fisioterapeutas. Apesar da amostra reduzida neste estudo, observou-se que os profissionais em cargos de chefia apresentaram menor suscetibilidade ao estresse, e maior naqueles que exerciam atividades assistenciais diretas.

Em relação ao modelo ERI, a preva-lência de alto esforço despendido para a execução do trabalho foi de $28,7 \%$, enquanto o baixo esforço alcançou o percentual de 39,4\%. Já para baixa recompensa recebida, a frequência foi de $33 \%$. Quanto à dimensão de compromisso excessivo com o trabalho, alto comprometimento foi relatado por 29,8\% (Tabela 4). 
Tabela 4

Frequência de esforço e recompensa pelos profissionais do Centro de Especialidades Médicas de Aracaju e do Centro de Especialidades Odontológicas, Aracaju, Sergipe, Brasil, 2011

\begin{tabular}{lcc}
\hline Modelo esforço-recompensa & $\mathbf{n}$ & $\%$ \\
\hline Esforço & 37 & 39,4 \\
$\quad$ Baixo & 30 & 31,9 \\
Médio & 27 & 28,7 \\
Alto & & \\
& & \\
Recompensa & 30 & 33,0 \\
Baixa & 31 & 34,0 \\
Média & 30 & 33,0 \\
Alta & & \\
Excesso de compromisso & & \\
Baixo & & 45,7 \\
Médio & 43 & 24,5 \\
Alto & 23 & 29,8 \\
\hline
\end{tabular}

Fonte: Os autores.

A análise de níveis de recompensa percebidos com relação à categoria profissional evidenciou que a baixa recompensa foi encontrada com maior frequência em psicólogos e terapeutas ocupacionais (80\%), seguindo-se assistentes sociais $(55,6 \%)$, fonoaudiólogos (50\%), fisioterapeutas $(37,5 \%)$ e enfermeiros $(33,3 \%)$. Os odontólogos e médicos apresentaram maior frequência de alta recompensa, respectivamente $50 \%$ e 47,8\% (Tabela 5).

Tabela 5

Frequência de recompensa (ERI) segundo categoria profissional no Centro de Especialidades Médicas de Aracaju e no Centro de Especialidades Odontológicas, Aracaju, Sergipe, Brasil, 2011

\begin{tabular}{lcccccc}
\hline & \multicolumn{2}{c}{ Recompensa global } & \multicolumn{2}{c}{ Alta } \\
Categoria profissional (91) & \multicolumn{2}{c}{ Baixa } & \multicolumn{2}{c}{ Média } & \multicolumn{2}{c}{ Alta } \\
& $\mathbf{n}$ & $\%$ & $\mathbf{n}$ & $\%$ & $\mathbf{n}$ & $\%$ \\
\hline Médicos & 11 & 44,0 & 11 & 44,0 & 11 & 44,0 \\
Enfermeiros & 10 & 55,6 & 10 & 55,6 & 10 & 55,6 \\
Odontólogos & 2 & 25,0 & 2 & 25,0 & 2 & 25,0 \\
Assistentes sociais & 4 & 50,0 & 4 & 50,0 & 4 & 50,0 \\
Fisioterapeutas & 4 & 57,1 & 4 & 57,1 & 4 & 57,1 \\
Fonoaudiólogos & 5 & 83,3 & 5 & 83,3 & 5 & 83,3 \\
Psicólogos & 4 & 80,0 & 4 & 80,0 & 4 & 80,0 \\
Terapeutas ocupacionais & 3 & 60,0 & 3 & 60,0 & 3 & 60,0 \\
Nutricionistas & 1 & 33,3 & 1 & 33,3 & 1 & 33,3 \\
Farmacêuticos & - & 0,0 & - & 0,0 & - & 0,0 \\
Biomédicos & 1 & 50,0 & 1 & 50,0 & 1 & 50,0 \\
\hline
\end{tabular}

Fonte: Os autores. 
Verificou-se associação entre alto esforço, baixa recompensa e compromisso excessivo de trabalho e prevalência de TMC. A prevalência de TMC foi de $74,1 \%$ entre os que apresentaram alto esforço no trabalho, 79,3\% entre aqueles com baixa recompensa e $82,1 \%$ entre os que possuíam condição de compromisso excessivo com suas atribuições ocupacionais. As diferenças observadas foram estatisticamente significantes ( $\mathrm{p}<0,05)$ (Tabela 6).

Todos os profissionais $(100,0 \%)$ cuja razão entre esforços e recompensas mostrou-se maior que 1 , indicando situação em que os esforços eram superiores às recompensas recebidas, foram positivamente classificados pelo SRQ-20 como suspeitos de terem um TMC. Ou seja, o desequilíbrio entre os esforços envolvidos na realização do trabalho e as recompensas que o trabalho oferece mostrou-se fortemente relacionado a efeitos negativos sobre a saúde mental dos profissionais estudados (Tabela 6). Fogaça e colaboradores (2009) realizaram um estudo transversal com 37 médicos e vinte enfermeiros com o objetivo de investigar as relações entre o trabalho e a qualidade de vida dos médicos e enfermeiros em unidades de terapia intensiva pediátrica e neonatal. Observaram que a presença de alto esforço e altas demandas psicológicas e físicas nesses profissionais repercutiam negativamente na sua qualidade de vida.

Tabela 6

Prevalência de transtornos mentais comuns (TMC), segundo o modelo esforço-recompensa (ERI), em profissionais do Centro de Especialidades Médicas de Aracaju e do Centro de Especialidades Odontológicas, Aracaju, Sergipe, Brasil, 2011

\begin{tabular}{|c|c|c|c|c|}
\hline \multirow{2}{*}{ Modelo esforço-recompensa } & \multirow[t]{2}{*}{$\mathrm{N}$} & \multicolumn{2}{|c|}{ TMC } & \multirow[t]{2}{*}{ Valor de $\mathrm{p}$} \\
\hline & & n & $\%$ & \\
\hline Esforço & 89 & & & \\
\hline Baixo & 33 & 7 & 21,2 & $<0,001$ \\
\hline Médio & 29 & 18 & 62,1 & \\
\hline Alto & 27 & 20 & 74,1 & \\
\hline Recompensa no trabalho & 86 & & & \\
\hline Baixa & 29 & 23 & 79,3 & 0,001 \\
\hline Média & 27 & 10 & 37,0 & \\
\hline Alta & 30 & 10 & 33,3 & \\
\hline Excesso de compromisso & 89 & & & \\
\hline Baixo & 40 & 11 & 27,5 & $<0,001$ \\
\hline Médio & 21 & 11 & 52,4 & \\
\hline Alto & 28 & 23 & 82,1 & \\
\hline Desequilíbrio esforço-recompensa & 88 & & & \\
\hline$<1$ & 72 & 26 & 38,8 & - \\
\hline$>1$ & 16 & 16 & 100,0 & \\
\hline
\end{tabular}

Fonte: Os autores. 
Situações estressoras, aqui avaliadas como alto esforço, baixa recompensa e compromisso excessivo com o trabalho, foram encontradas nos profissionais estudados. Quase um quinto dos trabalhadores $(18,2 \%)$ referiu haver um desequilíbrio entre os esforços envolvidos no trabalho e as recompensas recebidas. Knesebeck e colaboradores (2010), em seu estudo com cirurgiões na Alemanha, identificaram que cerca de um quarto dos 1.311 médicos pesquisados sofriam de desequilíbrio esforço-recompensa em seus trabalhos. Silva e Barreto (2010), após realizar um estudo transversal com 696 enfermeiros, técnicos e auxiliares de enfermagem de um hospital universitário, identificaram que $22 \%$ da população foi classificada como trabalhando em condições de desgaste e que $8 \%$ apresentavam mais esforços do que recompensas no trabalho.

Quando realizada análise de associação da relação esforço-recompensa e TMC, observou-se que quanto maior o esforço e o compromisso excessivo com o trabalho, maior a prevalência de TMC. No entanto, observou-se relação inversa com as recompensas no trabalho: quanto maiores as recompensas, menor a prevalência de TMC. Resultados similares de efeitos negativos de situações de desequilíbrio entre esforços e recompensas sobre a saúde têm sido observados em estudos com profissionais de saúde (Fogaça et al., 2009; Griep et al., 2011) e em outras categorias de trabalhadores, como os eletricitários (Souza et al., 2009).

Em relação à associação entre recompensa e categoria profissional, observou-se baixa recompensa em profissionais como psicólogos e terapeutas ocupacionais, seguidos de assistentes sociais, fonoaudiólogos e fisioterapeutas - em detrimento dos odontólogos e médicos, que apresentaram maior frequência no grupo que referiu alta recompensa.

No caso específico da remuneração e de carga horária semanal, pode-se destacar discrepâncias importantes entre as equipes de trabalho. De acordo com o decreto n. 3.382, de 28 de fevereiro de 2011 (Aracaju, 2011), os médicos e odontólogos possuem carga horária de vinte horas semanais, diferentemente das demais categorias, que trabalham trinta horas por semana. Além disso, profissionais como psicólogos, terapeutas ocupacionais, fonoaudiólogos, fisioterapeutas, nutricionistas e farmacêuticos recebem remuneração com valores de referência menores que as demais categorias, mesmo possuindo formação superior (Quadro 1). As diferenças salariais significativas e o tratamento diferenciado oferecido aos trabalhadores de formação superior de uma mesma unidade repercutem negativamente no bem-estar e na saúde do profissional.

Diversos fatores podem contribuir para a produção e a manutenção das diferenças de tratamento na equipe de saúde: características específicas do trabalho dos profissionais, condições de trabalho diferenciadas, dinâmica do mercado de trabalho, maior ou menor coesão interna dos profissionais 
com seus pares diretos, maior nível de organização de classe para a defesa de seus direitos e processo de valorização social das atividades desenvolvidas, incluindo-se a remuneração. De qualquer modo, é crucial para o adequado desempenho das ações e para a obtenção das metas estabelecidas de assistência, a construção de relações mais igualitárias entre os profissionais das equipes de saúde. Considerando que o modelo de oferta de atenção integral à população preconiza o atendimento pleno às múltiplas demandas dos pacientes e que, para que assim ocorra, é necessária a ação conjunta de diversos profissionais de saúde, devem ser feitos esforços no sentido de promoção de relações mais coesas e cooperativas entre os trabalhadores - o que realmente pode ser dificultado pelas diferenças de tratamento dos profissionais nas políticas públicas existentes e no modelo de gestão do trabalho adotado. Portanto, esse é um aspecto que merece maior reflexão.

Quadro 1

Carga horária de trabalho e níveis de referência de remuneração segundo a profissão, Aracaju, Sergipe, 2011

\begin{tabular}{|lcc|}
\hline Cargo/área & $\begin{array}{c}\text { Carga horária } \\
\text { (horas/semana) }\end{array}$ & $\begin{array}{c}\text { Valores de referência } \\
\text { R\$\$ }\end{array}$ \\
\hline Médico & 20 & $2.232,33$ \\
Médico & 30 & $3.348,49$ \\
Cirurgião-dentista & 20 & $1.800,86$ \\
Enfermeiro & 30 & $2.960,88$ \\
Assistente social & 30 & $2.485,65$ \\
Farmacêutico & 30 & $1.446,86$ \\
Fisioterapeuta & 30 & $1.446,86$ \\
Nutricionista & 30 & $1.446,86$ \\
Psicólogo clínico & 30 & $1.446,86$ \\
Terapeuta ocupacional & 30 & $1.446,86$ \\
Fonoaudiólogo & 30 & $1.446,86$ \\
\hline
\end{tabular}

Fonte: Os autores.

Segundo Areias e Comandule (2004), uma das dimensões que favorecem a qualidade de vida no trabalho é a econômica, representada pela equidade salarial, evitando-se a injustiça entre os profissionais. O Quadro 1 e as análises obtidas com base nas correlações entre satisfação salarial, satisfação com o trabalho, TMC e recompensa global, conforme as diferentes categorias, ilustram a situação de diferenças nas condições de trabalho dos serviços estudados, com possíveis impactos negativos sobre a saúde mental decorrentes desses processos diferenciados. 
Os resultados obtidos neste estudo devem ser avaliados, por sua vez, com cautela em razão de possíveis limitações. Estudos de corte transversais apresentam limitações como a impossibilidade no estabelecimento de causa e efeito, pois as medidas de exposição são identificadas no mesmo período. Além disso, houve baixa adesão de algumas categorias profissionais (médicos e odontólogos) e um quantitativo pouco representativo de algumas outras (nutricionistas, farmacêuticos e biomédicos). Tal fato dificultou a realização de comparações internas e com outros estudos. Além disso, os dados podem não oferecer um quadro fidedigno e completo do conjunto de trabalhadores dos serviços investigados - ou seja, a taxa de retorno dos questionários próxima à metade da população elegível é um fator que limita sobremaneira a extrapolação dos resultados obtidos. Registra-se, no entanto, que se obtiveram elevados percentuais de participação entre os profissionais que mais recentemente foram incorporados, de modo mais amplo, aos serviços públicos de saúde: $100 \%$ de participação entre assistentes sociais, fisioterapeutas, fonoaudiólogos e terapeutas ocupacionais; 83,3\% entre os psicólogos; e 66,6\% entre biomédicos. Portanto, o estudo parece expressar, com considerável fidedignidade, a situação vivenciada por esses profissionais nos centros estudados.

O pequeno número de pessoas estudado em algumas categorias profissionais exige cautela na interpretação das prevalências de TMC. Ou seja, quando se investiga um pequeno número de pessoas, a ocorrência de apenas um caso pode representar uma proporção muito elevada do evento. Então as comparações com outros estudos podem ficar comprometidas. Cabe destacar, no entanto, que de fato nas unidades estudadas, como nos serviços similares ofertados em outros locais, o número de profissionais em ocupações menos tradicionais no campo da saúde é, de modo geral, restrito, significando um percentual ainda pequeno de trabalhadores no conjunto das equipes. São ocupações de inserção mais recente nos serviços públicos de saúde, feitas a partir da criação do SUS. Portanto, ainda que tal panorama implique limitações muito claras nas comparações e extrapolação dos resultados obtidos, decorre de uma situação concreta, real. Os resultados aqui produzidos traduzem, ao menos parcialmente, uma dada situação e foram capazes de gerar informações úteis para a gestão do trabalho em saúde, constituindo uma base importante de informações para a elaboração de medidas de intervenção.

Outro fator de limitação desta pesquisa refere-se às dificuldades decorrentes dos estudos que utilizam questionários autoaplicáveis, em que os participantes podem não responder a todas as questões, possibilitando a perda de dados. Desse modo, os resultados aqui obtidos devem ser analisados com cuidado. 


\section{Conclusão}

Os resultados apresentados apontaram para expressiva prevalência de estresse ocupacional e transtornos mentais comuns entre os profissionais de saúde nos dois centros estudados, sendo especialmente elevada entre as categorias terapêuticas, mais envolvidas com as atividades de reabilitação. Fonoaudiólogos, psicólogos, terapeutas ocupacionais e fisioterapeutas estavam mais suscetíveis aos transtornos em relação às demais categorias estudadas.

Foram identificadas características do trabalho que apontaram condições desfavoráveis ao profissional, bem como ao desempenho das suas funções. Observou-se associação entre satisfação no trabalho, satisfação salarial e TMC. A alta prevalência de transtornos mentais comuns entre os profissionais de saúde investigados aponta a necessidade de ações com o intuito de proporcionar maior atenção à saúde mental desses trabalhadores, levando em consideração os aspectos psicossociais do trabalho. O desequilíbrio na relação entre esforço e recompensa mostrou-se importante fator associado a efeitos negativos sobre a saúde mental. Foi observada maior frequência de transtornos mentais comuns entre os profissionais com alto esforço, baixa recompensa e excessivo comprometimento com o trabalho.

Além disso, identificaram-se discrepâncias em relação à recompensa entre as diferentes categorias profissionais. Psicólogos, terapeutas ocupacionais, assistentes sociais, fonoaudiólogos e fisioterapeutas encontravam-se em situações desfavoráveis em relação às recompensas em comparação com outras categorias. Tal fato ratifica as relações de desigualdades no interior das unidades de saúde estudadas, evidenciando a necessidade de adoção de medidas que possam redesenhar essa situação, promovendo maior igualdade entre os profissionais. A característica de interdependência destes para a realização do adequado cuidado à saúde daqueles que dela necessitam impõe a necessidade de que níveis similares de tratamento, reconhecimento e valorização sejam os princípios norteadores das relações de trabalho no setor saúde. Políticas voltadas para essas ações devem, então, ser viabilizadas no município estudado.

Assim, merecem atenção especial as reflexões sobre condições de trabalho, incentivo às atividades cooperativas e mais coesas entre os profissionais das equipes e constituição de mecanismos que possam assegurar uma base mais integrada de ações e de sistemas mais igualitários de recompensas e reconhecimento. Nessa perspectiva, este estudo pode ser útil como indicador para adoção de medidas promotoras de saúde aos profissionais de saúde do Cemar e do CEO, no intuito de oferecer a eles maior qualidade de vida no seu trabalho e, consequentemente, um serviço de melhor qualidade à população. 


\section{Colaboradores}

Arthur Marcelino Filho participou da construção do projeto de pesquisa, da concepção e elaboração do artigo e de sua revisão; foi o responsável pelas atividades de coleta de dados (trabalho de campo) e análise dos dados. Tânia Maria de Araújo auxiliou em todas as etapas de realização do estudo (planejamento e execução), da construção do projeto de pesquisa à análise dos dados, da concepção e elaboração e revisão do manuscrito.

Resumen Las características ambientales y el modo de organización del trabajo en centros especializados en salud pueden generar situaciones de estrés y efectos negativos en la salud de los trabajadores. El objetivo del estudio fue evaluar la salud mental de los profesionales de la salud de la red especializada de Aracaju, Estado de Sergipe, Brasil, y las situaciones de estrés laboral, en base al modelo esfuerzo-recompensa. En 2011 se realizó un estudio transversal con 94 profesionales de la salud en actividad, en dos centros de atención especializada. Se utilizó un cuestionario conteniendo variables sociodemográficas, evaluación de estrés laboral (usando el Effort-Reward Imbalance) y el Self Reporting Questionaire, para medir los trastornos mentales comunes. La prevalencia de los trastornos mentales comunes fue elevada $(50,6 \%)$, y se mostró más frecuente en fonoaudiólogos, psicólogos, terapeutas ocupacionales y fisioterapeutas, y entre aquellos que relataron estar insatisfechos con su condición salarial y laboral. Todos los profesionales que hicieron referencia a desequilibrio entre esfuerzos y recompensas en el trabajo presentaron trastornos mentales comunes. En conclusión, se identificaron características del trabajo desfavorables a los profesionales y al desempeño de sus funciones, además de elevada prevalencia de trastornos mentales comunes. Deben implementarse programas orientados a la protección, valorización y promoción de la salud de los trabajadores, con el fin de elevar la calidad de vida en el trabajo.

Palabras clave estrés; centro de especialidades médicas, desordenes mentales, factores psicosociales del trabajo.

\section{Notas}

l Secretaria Municipal de Saúde de Aracaju, Sergipe, Brasil.

$<$ arthur_amf@yahoo.com.br>

Correspondência: Rua Duque de Caxias, 170, apartamento 101, São José, CEP 49015-320, Aracaju, Sergipe, Brasil.

2 Universidade Estadual de Feira de Santana, Feira de Santana, Bahia, Brasil.

<araujo.tania@uefs.br> 
3 Este artigo é resultado do trabalho de monografia intitulado Estresse Ocupacional e Saúde Mental dos profissionais do Centro de Especialidades Médicas de Aracaju, de autoria de Arthur Marcelino Filho e Tânia Maria de Araújo, apresentado no Curso de Especialização em Saúde Coletiva, com área de concentração em Gestão de Sistemas e Serviços de Saúde, realizado no Instituto de Saúde Coletiva da Universidade Federal da Bahia, 2011.

\section{Referências}

ARACAJU. Lei complementar n. 61, de 2 de julho de 2003. Institui o plano de carreiras, cargos e vencimentos para os servidores da categoria ocupacional da saúde do Município de Aracaju e dá outras providências. Palácio "Ignácio Barbosa", Aracaju, 2 jul. 2003.

ARACAJU. Decreto n. 3.382, de 28 de fevereiro de 2011. Regulamenta os artigos 44, 44-A e 44-B da lei complementar n. 61, de 2 de julho de 2003, alterada por leis posteriores Centro Administrativo "Prefeito Aloísio Campos", Aracaju, 28 fev. 2011.

ARAÚJO, Tânia. M.; ROTENBERG, Lúcia. Relações de gênero no trabalho em saúde: a divisão sexual do trabalho e a saúde dos trabalhadores. In: ASSUNÇÃO, Ada A.; BRITO, Jussara (orgs.). Trabalhar na saúde: experiências cotidianas e desafios para a gestão do trabalho e do emprego. Rio de Janeiro: Editora Fiocruz, 2011. p. 131-159.

ARAÚJO, Tânia M. et al. Aspectos psicossociais do trabalho e distúrbios psíquicos entre trabalhadoras de enfermagem. Revista de Saúde Pública, São Paulo, v. 37. n. 4, p. 424-433, 2003. Disponível em: <www. scielo.br/pdf/rsp/v37n4/16776.pdf $>$. Acesso em: 20 jun. 2012.

ARAÚJO, Tânia M. et al. Transtornos mentais comuns em mulheres: estudo comparativo entre donas de casa e trabalhadoras. Revista de Enfermagem Uerj, Rio de Janeiro v. 149, n. 2, p. 260-269, 2006. Disponível em: $<$ www.facenf.uerj.br/v14n2/v14n2a17.pdf $>$. Acesso em: 20 jun. 2012.

AREIAS, Maria E. Q.; COMANDULE, Alexandre. Q. Qualidade de vida, estresse no trabalho e síndrome de burnout. VILARTA, R.; CARVALHO, T., GONÇALVES, A., \& GUTIERREZ, G. Qualidade de vida e fadiga institucional. Campinas: IPES Editorial, 2006. p. 183-202.

CABANA, Maria C. F. L. et al. Transtornos mentais comuns em médicos e seu cotidiano de trabalho. Jornal Brasileiro de Psiquiatria, Rio de Janeiro, v. 569, n. 1, p. 33-40, 2007. Disponível em: <www.scielo.br/scielo.php? pid=S0047-20852007000100009\&script $=$ sci_arttext>. Acesso em: 20 jun. 2012.

CHOR, Dóra et al. The Brazilian version of the Effort-Reward Imbalance questionnaire to assess job stress. Cadernos de Saúde Pública, Rio de Janeiro, v. 24. n. 1, p. 219-224, jan. 2008. Disponível em: <www.scielo.br/ pdf/ csp/v24nl/21.pdf>. Acesso em: 19 jun. 2012.

DE CARVALHO, Liliane; MALAGRIS, Lucia Emmanoel N. Avaliação do nível de stress em profissionais de saúde. Estudos e pesquisas em psicologia, Rio de Janeiro, v. 7, n. 3, p. 570-582, 2007.

DELCOR, Núria S. et al. Condições de trabalho e saúde dos professores da rede particular de ensino de Vitória da Conquista, 
Bahia, Brasil. Cadernos de Saúde Pública, Rio de Janeiro, v. 20, n. 1, p. 187-196, 2004. Disponível em: <www.scielo.br/pdf/csp/ v20nl/35.pdf $>$. Acesso em: 20 jun. 2012.

DE MARCO, Patrícia F. et al. O impacto do trabalho em saúde mental: transtornos psiquiátricos menores, qualidade de vida e satisfação profissional. Revista Brasileira de Psiquiatria, São Paulo, v. 57, n. 3, p. 178-183, 2008. Disponível em: <www.scielo.br/pdf/ jbpsiq/v57n3/04.pdf>. Acesso em: 18 jun. 2012.

FERNANDEZ-LOPEZ, Juan A. et al. El trabajo y sus repercusiones em la salud: el modelo "Desequilibrio-Esfuerzo-Recompensa-DER". Revista Calidad Asistencial, Oviedo, v. 20, n. 3, p. 165-170, 2005.

FOGAÇA, Monalisa de C. et al. Estresse ocupacional e suas repercussões na qualidade de vida de médicos e enfermeiros intensivistas pediátricos e neonatais. Revista Brasileira de Terapia Intensiva, São Paulo, v. 21, n. 3, p. 299-305, 2009.

FRANCO, Tulio B.; MAGALHÃES JR., Helvécio $M$. A integralidade na assistência da saúde: a organização das linhas de cuidado. In: MERHY, E. et al. O trabalho em saúde: olhando e experienciando o SUS no cotidiano. São Paulo: Hucitec, 2004. p. 125-133.

GRIEP, Rosane R. et al. Uso combinado de modelos de estresse no trabalho e a saúde autorreferida na Enfermagem. Revista de Saúde Pública, São Paulo, v. 45, n. 1, p. 145-152, 2011. Disponível em: <www.scielo.br/scielo. php? script $=$ sci_arttext $\&$ pid $=$ S0034-89102 $011000100017>$. Acesso em: 20 jun. 2012.

GUIMARÃES, José M. X. et al. (In)satisfação com o trabalho em saúde mental: um estudo em centros de atenção psicossocial. Ciência \& Saúde Coletiva, Rio de Janeiro, v. 16, n. 4, p. 2.145-2.154, 2011.

VON DEM KNESEBECK, Olaf et al. Psychosocial stress among hospital doctors in surgical fields: results of a nationwide survey in Germany. Deutsches Arzteblatt International, Colônia, 107, n. 14, p. 248, 2010.
LEITE, Marcia. Reestruturação produtiva e mercado de trabalho: a experiência brasileira. Revista Galena de Economia, Santiago de Compostela, v. 1-2, n. 14, p. 1-26, 2005.

LI, Jian et al. Effort-Reward Imbalance at work and job dissatisfaction in Chinese healthcare workers: a validation study. International Archives Occupational Environmental Health, Berlim, v. 78, n. 3, p. 198-204, 2005.

MACHADO, Jorge H.; ASSUNÇÃO, Ada A. Panorama da saúde dos trabalhadores da saúde. Belo Horizonte: Faculdade de Medicina/Departamento de Medicina Preventiva e Social, UFMG, 2012. 164 p.

MAGALHÃES, Andrea B. A sindrome de Burnout no contexto hospitalar pediátrico. $146 \mathrm{f}$. Dissertação (Mestrado em Psicologia) - Universidade Federal de Goiás, Goiânia, 2006.

MARI Jair J.; WILLIAMS, Paul. A validity study of a psychiatric screening questionnaire (SRQ-20) in primary care in the city of São Paulo. British Journal of Psychiatry. Londres, v. 148, p. 23-26, 1986.

NAKAO, Mutsuhiro. Work-related stress and Psychosamatic Medicine. BioPsychoSocial Medicine, Londres, v. 4, n. 1, p. 4-12, 2010.

NASCIMENTO SOBRINHO, Carlito L. et al. Condições de trabalho e saúde dos médicos em Salvador, Brasil. Revista da Associação Médica Brasileira, São Paulo, v. 52, n. 2, p. 97-102, 2006.

REIS, Eduardo J. F. B. et al. Trabalho e distúrbios psíquicos em professores da rede municipal de Vitória da Conquista, Bahia, Brasil. Cadernos de Saúde Pública, Rio de Janeiro, n. 20, v. 1, p. 1.480-1.496, 2005.

SANTOS, Kionna O. et al. Estrutura fatorial e consistência interna do Self-Reporting Questionnaire (SRQ-20) em população urbana. Cadernos de Saúde Pública, Rio de Janeiro, v. 25, n. 1, p. 214-222, jan. 2009.

SANZOVO, Cristiane E.; COELHO, Myrna E. C. Estressores e estratégias de coping em 
uma amostra de psicólogos clínicos. Estudos de Psicologia, Campinas, v. 24, n. 2, p. 227238, 2007.

SIEGRIST, Johannes et al. The measurement of Effort-Reward Imbalance at work: European comparisons. Social Science \& Medicine, Amsterdam, v. 58 , n. 8, p. $1.483-$ 1.499, 2004.

SIEGRIST, Johannes. Effort-Reward Imbalance at work and cardiovascular diseases. International Journal of Occupational Medicine and Environmental Health, Berlim, v. 23, n. 3, p. 279-285, 2010.

SILVA, Luiz S.; BARRETO, Sandhi M. Adaptação transcultural para o português brasileiro da escala Effort-Reward Imbalance: um estudo com trabalhadores de banco. Revista de Saúde Pública, São Paulo, v. 44, n. 4, p. 718-725, 2010.

SIQUEIRA, Carlos G. A.; PEDRUZZI, Cristiane M. Trabalho e saúde: percepções e significados para a fonoaudiologia. Distúrbios da Comunicação, São Paulo, v. 21, n. 2, p. 219-228, 2009.
SOUZA, Magda N. M.; MARTINS-JÚNIOR, Davi F.; SILVA, Marina V. Trabalho e saúde dos profissionais de enfermagem de um hospital especializado de Feira de Santana, Bahia. Revista Baiana de Saúde Pública, Salvador, v. 35, p. 38, 2011.

SOUZA, Suerda F. et al. Trabalho e saúde mental dos trabalhadores de manutenção de um sistema de geração e transmissão de energia elétrica. 164 f. Dissertação (Mestrado em Saúde, Ambiente e Trabalho) - Universidade Federal da Bahia, Salvador, 2009.

VASCONCELOS, Eveli F.; GUIMARÃES, Liliana A. M. Esforço e recompensa no trabalho de uma mostra de profissionais de Enfermagem. Psicólogo in Formação, São Paulo, ano 13, n. 13, jan./dez. 2009. Disponível em: <www.metodista.br/revistas/ revistas-ms/index.php/PINFOR/article/ viewFile/2075/2040> . Acesso em: 17 fev. 2014.

Recebido em 26/11/2012

Aprovado em 08/03/2014 